\title{
Reduced-Complexity Iterative Receiver for Improving the IEEE 802.15.7 Convolutional-Coded Color Shift Keying Mode
}

\author{
Zunaira Babar, Chuan Zhu, Hung Viet Nguyen, Panagiotis Botsinis, Dimitrios Alanis, \\ Daryus Chandra, Soon Xin Ng and Lajos Hanzo
}

\begin{abstract}
In this paper, we conceive novel symbol-based ColorShift Keying (CSK)-aided concatenated coding schemes, which provide attractive performance gains over the comparable bitbased systems. Quantitatively, our 4CSK-aided and 16CSK-aided symbol-based concatenated systems require $0.8 \mathrm{~dB}$ and $0.45 \mathbf{d B}$ lower SNR than the equivalent bit-based schemes. In terms of decoding complexity, the 4CSK-aided and 16CSK-aided systems reduce the decoding complexity by $67 \%$ and $33 \%$, respectively. We have also analyzed the convergence behavior of our system with the aid of non-binary EXtrinsic Information Transfer (EXIT) charts adapted for symbol-based iterative CSK-assisted systems.
\end{abstract}

Keywords-Visible Light Communication, Color-Shift Keying, Iterative Decoding, EXIT charts.

\section{INTRODUCTION}

Visible Light Communication (VLC) [1], [2] has emerged as a promising alternative to the conventional Radio Frequency (RF)-based wireless systems, since it harnesses the license-free visible light band. Explicitly, VLC exploits the existing lighting infrastructure for communication by intensity modulating the light sources at high speeds. Consequently, data rates of several Gigabits per second may be achieved over short distances.

The availability of multi-colored LEDs in VLC systems has led to the development of Color-Shift Keying (CSK), which is standardized in IEEE 802.15.7 [1] for achieving high data transmission rates. The research fraternity has primarily focused on optimizing the CSK constellations [3]-[5]. However, recently a soft-decision aided CSK detector was conceived [6] for iterative CSK transceivers, while CSK aided unequal error protection schemes were developed in [7] for video applications. The concatenated CSK-aided code designs of [6] invoke bit-based iterative decoding, which either requires improved bit-to-symbol mapping strategies or a three-stage concatenated system for achieving a vanishingly low Bit Error Ratio (BER). To circumvent these issues, in this paper we focus our attention on designing symbol-based iterative CSKaided coding schemes. Explicitly, our main contributions are:

- We quantify the symbol-to-bit conversion loss associated with the standardized IEEE CSK constellations and

The authors are with the School of Electronics and Computer Science, University of Southampton, SO17 1BJ, United Kingdom. Email: \{zb2g10, hvn08r, pb1y14, da4g11, dc2n14, sxn, lh\}@ecs.soton.ac.uk.

The financial support of the European Research Council under the Advanced Fellow Grant, that of the Royal Society's Wolfson Research Merit Award and that of the Engineering and Physical Sciences Research Council under Grant $\mathrm{EP} / \mathrm{L} 018659 / 1$ is gratefully acknowledged. The research data for this paper is available at http://doi.org/10.5258/SOTON/D0088.

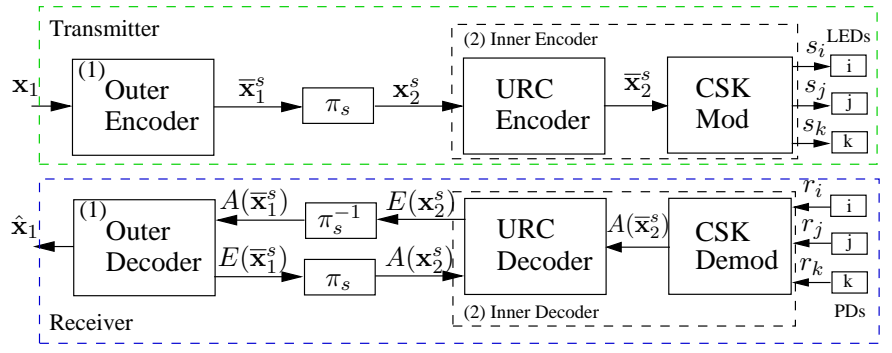

Fig. 1: Symbol-based $M$-ary CSK-aided VLC system.

conceive symbol-based iterative schemes for alleviating the loss. Our schemes provide beneficial gains over their bit-based counterparts both in terms of the achievable BER performance as well as the decoding complexity imposed.

- The conventional non-binary EXtrinsic Information Transfer (EXIT) charts of [8] do not accurately model the convergence behavior of symbol-based CSK-aided concatenated systems. Hence, we have carried out nonbinary EXIT chart analysis by modeling the associated a-priori probabilities using $M$-ary CSK symbols. Our results demonstrate that despite the specific nature of the triangular CSK constellation, our EXIT chart predictions closely match the Monte-Carlo simulations.

This letter is structured as follows. In Section II, we detail our system model, followed by a discussion on non-binary EXIT charts in Section III. Our results are discussed in Section IV, while our conclusions are offered in Section V.

\section{SYSTEM MODEL}

Fig. 1 shows the general schematic of a VLC transceiver relying on a symbol-based concatenated CSK-aided coding scheme. Explicitly, any non-binary Forward Error Correction (FEC) code may be employed as the outer decoder component. We propose to intrinsically amalgamate a recursive non-binary Unity Rate Code (URC) [9] with the CSK modulator to create a composite inner decoder component.

At the transmitter, the information bits $\mathbf{x}_{1}$ are encoded into the $M$-ary symbols $\overline{\mathbf{x}}_{1}^{s}$ by the outer encoder of Fig. 1. The encoded non-binary symbols are interleaved by a symbol interleaver $\left(\pi_{s}\right)$ and the interleaved symbols $\mathbf{x}_{2}^{s}$ are fed to a non-binary URC encoder having the generator polynomial of $G(D)=\frac{1}{1+D}$. The URC acts as a precoder, or rather as a scrambler, having an infinite impulse response, which 
beneficially spreads the extrinsic information without affecting the overall system throughput (or delay). Finally, the precoded stream $\overline{\mathbf{x}}_{2}^{s}$ is mapped onto $M$-ary CSK symbols, which are modulated using a combination of three distinct LEDs, having different wavelengths/frequencies. To elaborate further, each CSK symbol is defined as a point $\left(x_{m}, y_{m}\right)$ in the 2D color space, which is generated using three light sources denoted by $i, j$ and $k$. Consequently, the $x y$ color coordinates are mapped onto the equivalent optical intensity vector $\mathbf{s}=\left[s_{i}, s_{j}, s_{k}\right]^{T}$, where $s_{p}$ represents the power of the $p$ th light source.

At the receiver, each of the three optical signals are independently received by a separate Photo Detector (PD), which converts the received optical signals to electrical signals. Assuming lossless conversion from the optical to the electrical domain, the noisy received signal $\mathbf{r}=\left[r_{i}, r_{j}, r_{k}\right]^{T}$ can be represented as:

$$
\mathbf{r}=\mathbf{H} \mathbf{s}+\mathbf{n},
$$

where $\mathbf{H}$ is a $(3 \times 3)$-element matrix, whose diagonal entries specify the channel gain of each color, while all the other entries represent the interference imposed by the other colors. Furthermore, $\mathbf{n}=\left[n_{i}, n_{j}, n_{k}\right]^{T}$ characterizes the electrical AWGN noise, having a total variance of $\sigma^{2}$, where the noise variance at each PD is $\sigma_{0}^{2}=\sigma^{2} / 3$. In this paper, we consider a non-dispersive AWGN channel [6], [7], hence $\mathbf{H}$ is equivalent to the identity matrix. Furthermore, we define the electrical SNR as $\gamma_{e}=E_{s} / N_{0}$, where $E_{s}=\mathbb{E}\left\{\|\mathbf{s}\|^{2}\right\}$, while $N_{0}$ is the total electrical noise power. Here $\mathbb{E}$ denotes the expectation operation, while $\|$.$\| represents the Euclidean norm.$

Based on the received signal of Eq. (1), the CSK demodulator of Fig. 1 computes the soft a-priori probabilities $A\left(\overline{\mathbf{x}}_{2}^{s}\right)$ as follows:

$A\left(\overline{\mathbf{x}}_{2}^{s}=m\right) \approx P\left(\mathbf{r} \mid \mathbf{s}=\mathbf{s}^{(m)}\right)=\frac{1}{2 \pi \sigma_{0}} \exp \left(-\frac{\left\|\mathbf{r}-\mathbf{H s}^{(m)}\right\|^{2}}{2 \sigma_{0}^{2}}\right)$

where $\mathbf{s}^{(m)}$ is the $m$ th hypothetical $M$-ary CSK symbol for $m \in\{0, \ldots, M-1\}$ and we have assumed that all $M$ ary CSK symbols are equiprobable. The soft information of Eq. (2) is then fed to the URC decoder, which engages in iterative decoding with the outer decoder, as encapsulated in Fig. 1. Here the notations $A($.$) and E($.$) denote the a-priori$ and extrinsic probabilities, respectively.

Our concatenated design of Fig. 1 invokes a symbol interleaver. By contrast, the bit-based concatenated design of [6] invokes a bit interleaver, which in turn requires symbol-to-bit conversion at the output of the CSK demodulator. Explicitly, the symbol-based probabilities of Eq. (2) are marginalized to yield the corresponding bit-based probabilities. Since the bits within a CSK symbol are correlated, the symbol-to-bit conversion results in information loss, as demonstrated in Fig. $2^{1}$. This information loss may be mitigated by invoking an iterative CSK detector. However, as demonstrated in [6], the standard CSK detector does not exhibit good iterative capabilities. Consequently, the designs of [6] relied either on improved signal labeling strategies or on a three-stage concatenated architecture. As an alternative strategy, our system of Fig. 1

\footnotetext{
${ }^{1}$ Non-iterative CSK detector is considered in Fig. 2, whose capacity is the average mutual information between $\overline{\mathbf{x}}_{2}^{s}$ and $A\left(\overline{\mathbf{x}}_{2}^{s}\right)$ computed using Eq. (5).
}

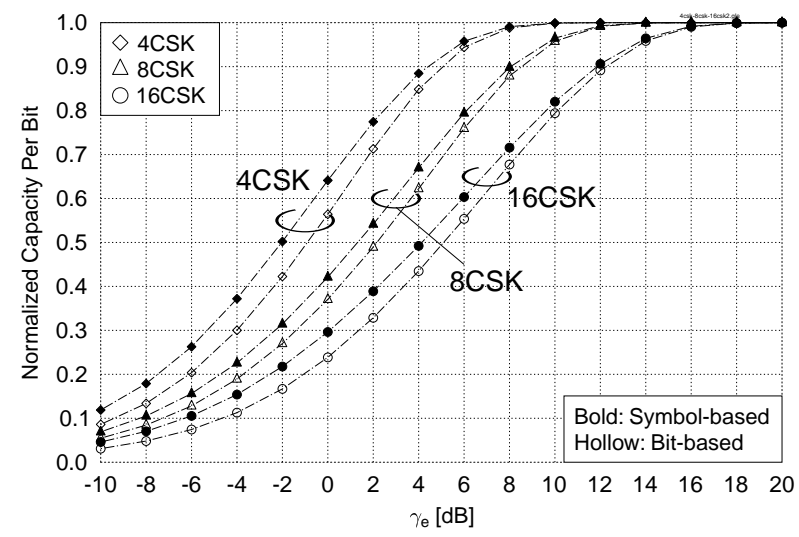

Fig. 2: Normalized symbol-based and bit-based capacities. Please note that the capacities are normalized with respect to the number of bits transmitted per symbol, so that the maximum achievable capacity is unity for all instances.

relies on the symbol-based probabilities for circumventing the symbol-to-bit conversion loss.

\section{NON-BINARY EXIT CHARTS FOR CSK-AIDED SYSTEMS}

For the sake of predicting the convergence behavior of the CSK-aided concatenated system of Fig. 1, we invoke a non-binary EXIT chart [8], which is a semi-analytical tool conceived for visualizing the exchange of average Mutual Information (MI) between the inner and outer decoders. Explicitly, $I_{A}\left(\mathbf{x}_{2}^{s}\right)$ and $I_{E}\left(\mathbf{x}_{2}^{s}\right)$ constitute the inner decoder's EXIT curve, while $I_{A}\left(\overline{\mathbf{x}}_{1}^{s}\right)$ and $I_{E}\left(\overline{\mathbf{x}}_{1}^{s}\right)$ constitute the outer decoder's EXIT curve, where $I_{A}(\mathbf{z})$ (or $I_{E}(\mathbf{z})$ ) is defined as the average MI between $\mathbf{z}$ and $A(\mathbf{z})$ (or $E(\mathbf{z})$, respectively) for $\mathbf{z} \in\left\{\overline{\mathbf{x}}_{1}^{s}, \mathbf{x}_{2}^{s}\right\}$.

In the conventional EXIT charts of [8], the $M$-ary a-priori probabilities $A(\mathbf{z})$ are modeled assuming that the bits within the $M$-ary symbol are independent and that they exhibit a Gaussian distribution. This assumption generally holds for bitbased systems only, since the symbol-based iterative schemes preserve the correlation of bits within a symbol [10]. Hence, we model the $M$-ary a-priori probabilities based on the transmission of $M$-ary CSK symbols over an AWGN channel, which can be formulated as:

$$
I_{A}(\mathbf{z})=I(\mathbf{s}, \mathbf{r}) \equiv J\left(\sigma_{A}^{2}\right),
$$

where $\mathbf{s}=\mu(\mathbf{z})$ for the $M$-ary CSK mapper $\mu$, while $\mathbf{r}=$ $\mathbf{s}+\mathbf{n}$. Furthermore, $I(\mathbf{s}, \mathbf{r})$ represents the normalized $M$-CSK channel capacity of Fig. 2, assuming that all CSK symbols are equiprobable, and $J\left(\sigma_{A}^{2}\right)$ is a monotonically decreasing function of $\sigma_{A}^{2}$. More specifically, for a given value of the $a$ priori MI $I_{A}(\mathbf{z})=b$, where $b \in[0,1]$, we model the received signal $\mathbf{r}$ using the channel noise $\mathbf{n}$, whose variance is:

$$
\sigma_{A}^{2}=J^{-1}\left[I_{A}(\mathbf{z})=b\right] .
$$

The resultant a-priori probabilities $A(\mathbf{z})$ may then be computed using Eq. (2). This should not be confused with the $a$ priori probabilities $A\left(\overline{\mathbf{x}}_{2}^{s}\right)$, which are received from the CSK demodulator of Fig. 1. It is important to highlight here that 
the symbols in $M$-CSK constellation are not equally spaced due to its triangular structure. Nevertheless, as it will be experimentally demonstrated by our results in Section IV, our Monte Carlo simulations closely follow the EXIT chart predictions.

The resultant average extrinsic MI of the inner and outer decoders can be formulated as [8]:

$$
I_{E}(\mathbf{z})=\log _{2} M+\mathbb{E}\left[\sum_{m=0}^{M-1} E(\mathbf{z}=m) \log _{2} E(\mathbf{z}=m)\right] .
$$

Finally, the inner and the inverted outer decoders' EXIT curves are plotted in the same graph. Hence, the exchange of MI between the two components may be visualized as a staircase-shaped trajectory. The SNR at which a marginally open EXIT-chart tunnel is attained defines the convergence threshold of the concatenated scheme.

\section{REsults AND Discussions}

In this section we benchmark the performance of our symbol-based scheme of Fig. 1 against the equivalent bit-based design and evaluate the accuracy of our EXIT chart analysis. The bit-based counterpart is obtained by replacing the symbol interleaver of Fig. 1 with a bit interleaver. Furthermore, we have used the standard CSK constellation of [1] for color band combination $(110,010,000)$, which transmits using red, yellow and blue colors.

As a first design example, we conceive a 4CSK-aided system, where the memory-3 1/2-rate Recursive Systematic Convolutional (RSC) code $(2,1,3)$ having octal generator polynomials $(15,13)_{8}$ constitutes the outer component; hence, it is referred to as the RSC-URC-4CSK arrangement. The EXIT characteristics of the system are plotted in Fig. 3. We may observe that the convergence threshold of the symbolbased scheme emerges around $\gamma_{e}=-1 \mathrm{~dB}$. By contrast, the inner and outer decoders' EXIT curves of the bit-based design cross-over at this SNR, hence closing the tunnel. Explicitly, the bit-based scheme requires a $0.75 \mathrm{~dB}$ higher SNR to yield a marginally open tunnel, which permits the decoding trajectories to reach the $(1,1)$-point of near-perfect convergence. The decoding trajectory of the symbol-based scheme is also plotted in Fig. 3 for a frame length of 10,000 symbols and $\gamma_{e}=-1 \mathrm{~dB}$, which closely follows the EXIT curves. The BER performance of the symbol-based RSC-URC-4CSK scheme is recorded in Fig. 4 for a frame length of 10,000 symbols. As it can be observed, the turbo-cliff formulates around $\gamma_{e}=-1 \mathrm{~dB}$, since the performance improves rapidly for $\gamma_{e} \geq$ $-1 \mathrm{~dB}$ as the number of iterations increases. This is in line with the convergence threshold predicted by our EXIT chart of Fig. 3. We have benchmarked the performance of our symbolbased scheme against that of the equivalent bit-based design after a maximum of 15 iterations. It may be observed that the symbol-based design outperforms its bit-based counterpart. Explicitly, at a BER of $10^{-5}$, the symbol-based design operates within $1.3 \mathrm{~dB}$ of the capacity after 15 iterations, while the bitbased scheme exhibits a deviation of $2.1 \mathrm{~dB}$ from the capacity, hence requiring around $0.8 \mathrm{~dB}$ higher SNR. We may also observe in Fig. 4 that only 5 decoding iterations are required by the symbol-based scheme to outperform the bit-based scheme

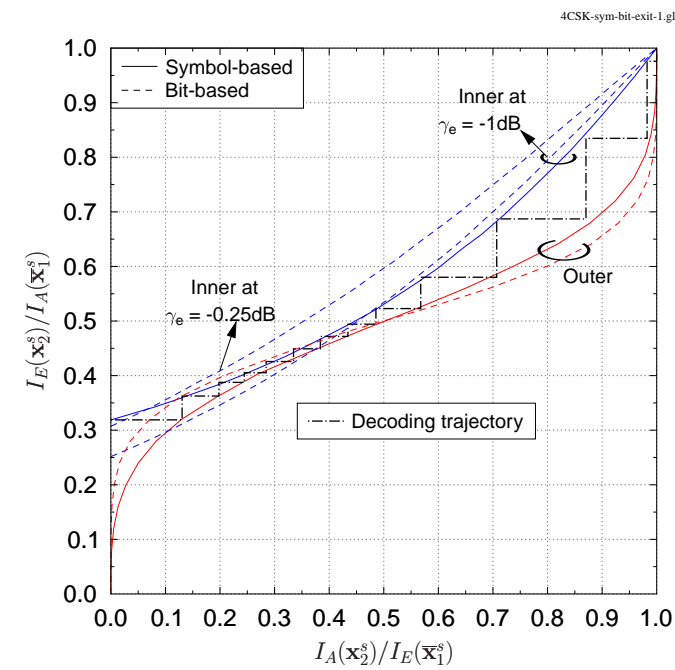

Fig. 3: Normalized EXIT curves of the symbol-based and bitbased RSC-URC-4CSK schemes. Decoding trajectory is also plotted for the symbol-based system at $\gamma_{e}=-1 \mathrm{~dB}$.

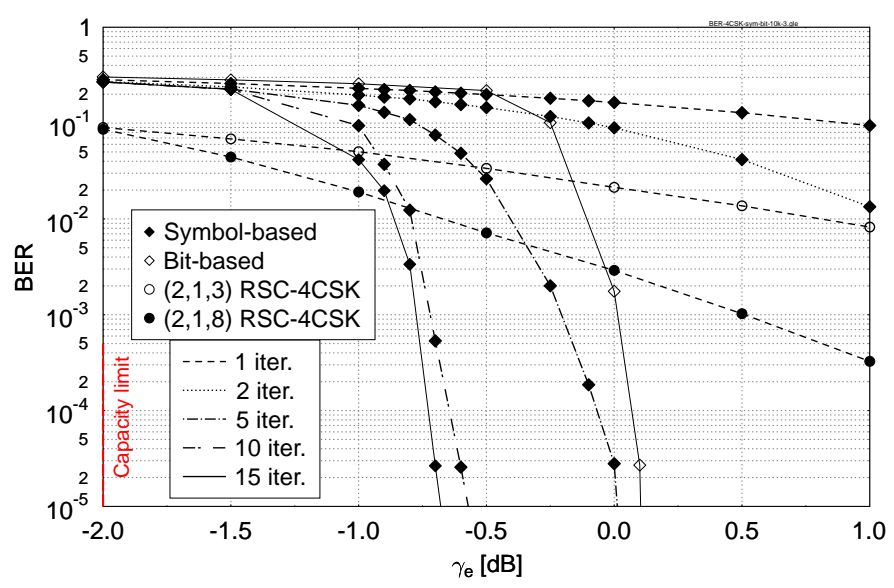

Fig. 4: BER performance of the symbol-based RSC-URC4CSK design upon increasing the number of iterations. The performance of bit-based counterpart after 15 iterations and that of the non-iterative RSC-4CSK schemes is also plotted for comparison. Frame length $=10,000$ symbols.

that invokes 15 iterations. Hence, our symbol-based RSCURC-4CSK scheme requires $67 \%$ less decoding iterations. We have also recorded the performance of non-iterative RSC4CSK schemes relying both on the $(2,1,3)$ and on the $(2,1,8)$ RSCs. We have used a memory- 8 RSC as our benchmark because it exhibits a higher decoding complexity than the RSC-URC-4CSK schemes. Explicitly, both the symbol-based as well as the bit-based RSC-URC-4CSK schemes invoke $\left(2^{3}+2^{1}\right)=10$ trellis states per iteration, hence invoking a total of 150 states during 15 iterations, which is less than the 256 states invoked by the $(2,1,8)$ RSC. Furthermore, we have used the generators $(561,753)_{8}$ for the $(2,1,8)$ RSC, which result in the maximum free distance. Both iterative schemes significantly outperform the non-iterative ones.

As our second design example, we have analyzed the performance of a RSC-URC-16CSK scheme in Fig. 5. Specif- 


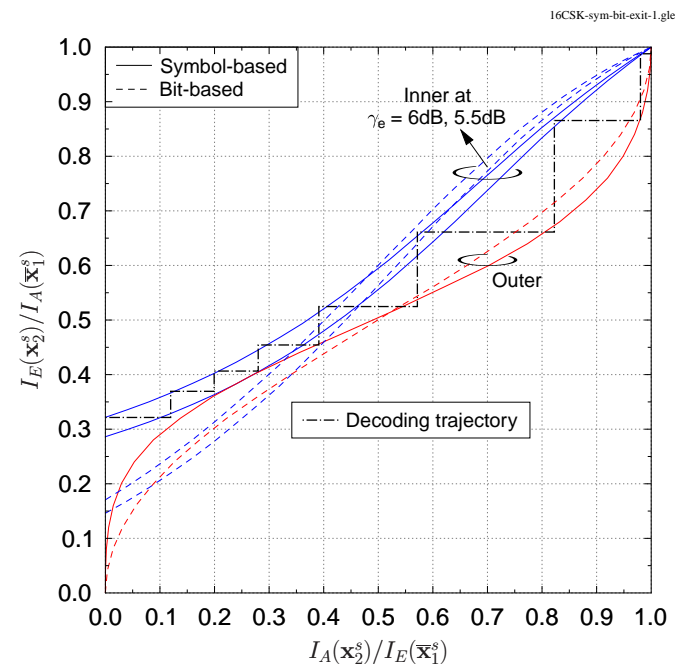

Fig. 5: Normalized EXIT curves of the symbol-based and bitbased RSC-URC-16CSK schemes. Decoding trajectory is also plotted for the symbol-based system at $\gamma_{e}=6 \mathrm{~dB}$.

ically, our RSC-URC-16CSK arrangement invokes a $(4,2,6)$ RSC as the outer component, which has the generators $(11,0,2,0 ; 0,11,0,2)_{8}$. Again, our symbol-based design exhibits a lower convergence threshold than the bit-based system and the decoding trajectory of the symbol-based system matches the inner and outer decoders' EXIT curves. Explicitly, the convergence threshold of the symbol-based system is around $\gamma_{e}=5.5 \mathrm{~dB}$, while that of the bit-based scheme is $\gamma_{e}=6 \mathrm{~dB}$. We have plotted the resultant BER performance curves in Fig. 6, which demonstrate that the symbol-based arrangement operates within $1.7 \mathrm{~dB}$ of the capacity at a BER of $10^{-5}$ and after 15 iterations, while the bit-based system is $2.15 \mathrm{~dB}$ away from the capacity limit. Furthermore, the symbol-based system requires around 10 iterations to outperform the bit-based system that employs 15 iterations. Hence, the decoding complexity is reduced by about $33 \%$. Finally, we may also observe in Fig. 6 that the bit-based and symbol-based RSC-URC-16CSK schemes exhibit a sharp turbo cliff, hence resulting in a much lower BER than the non-iterative RSC16CSK schemes, relying on the $(4,2,6)$ and $(2,1,10)$ RSCs. The $(2,1,10)$ RSC relies on the generators $(2335,3661)$, which maximize the free distance. Furthermore, it invokes 1024 trellis states during decoding, which is comparable to the 1020 states invoked by the RSC-URC-16CSK schemes during 15 iterations.

\section{Conclusions}

In this paper, we have proposed symbol-based concatenated coding schemes for CSK transmission for the sake of eliminating the information loss inherent in the bit-based systems. We have also adapted the conventional non-binary EXIT charts for analyzing the convergence behaviour of our non-binary system, where the bits of a symbol exhibit significant correlation. Our results demonstrate that the symbol-based coding schemes outperform their bit-based counterparts both in terms of the achievable BER performance as well as the decoding complexity imposed. Furthermore, our EXIT chart predictions are

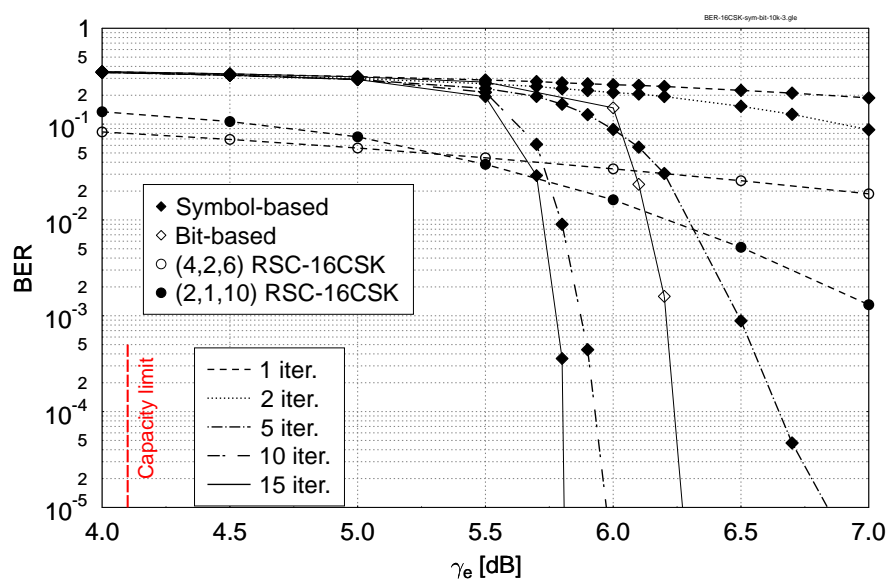

Fig. 6: BER performance of the symbol-based RSC-URC16CSK design upon increasing the number of iterations. The performance of bit-based counterpart after 15 iterations and that of the non-iterative RSC-16CSK schemes is also plotted for comparison. Frame length $=10,000$ symbols.

in conformity with the achievable BER performance, despite the specific nature of the triangular CSK constellation.

\section{REFERENCES}

[1] "IEEE standard for local and metropolitan area networks-part 15.7: Short-range wireless optical communication using visible light," IEEE Std 802.15.7-2011, pp. 1-309, Sept 2011.

[2] S. H. Chen and C. W. Chow, "Color-shift keying and code-division multiple-access transmission for RGB-LED visible light communications using mobile phone camera," IEEE Photonics Journal, vol. 6, no. 6, pp. 1-6, Dec 2014.

[3] E. Monteiro and S. Hranilovic, "Design and implementation of colorshift keying for visible light communications," Journal of Lightwave Technology, vol. 32, no. 10, pp. 2053-2060, May 2014.

[4] R. J. Drost and B. M. Sadler, "Constellation design for channel precompensation in multi-wavelength visible light communications," IEEE Transactions on Communications, vol. 62, no. 6, pp. 1995-2005, June 2014.

[5] R. Singh, T. O. Farrell, and J. P. R. David, "An enhanced color shift keying modulation scheme for high-speed wireless visible light communications," Journal of Lightwave Technology, vol. 32, no. 14, pp. 2582-2592, July 2014.

[6] J. Jiang, R. Zhang, and L. Hanzo, "Analysis and design of threestage concatenated color-shift keying," IEEE Transactions on Vehicular Technology, vol. 64, no. 11, pp. 5126-5136, Nov 2015.

[7] C. Zhu, Y. Huo, J. Jiang, H. Sun, C. Dong, R. Zhang, and L. Hanzo, "Hierarchical colour-shift-keying aided layered video streaming for the visible light downlink," IEEE Access, vol. 4, pp. 3127-3152, 2016.

[8] J. Kliewer, S. X. Ng, and L. Hanzo, "Efficient computation of EXIT functions for non-binary iterative decoding," IEEE Transactions on Communications, vol. 54, no. 12, pp. 2133-2136, December 2006.

[9] D. Divsalar, S. Dolinar and F. Pollara, "Serial concatenated trellis coded modulation with rate-1 inner code," in Proc. IEEE Global Telecommun. Conf., San Francisco, CA, Nov 2000, pp. 777-782.

[10] S. X. Ng, J. Y. Chung, and L. Hanzo, “Turbo-detected unequal protection MPEG-4 wireless video telephony using multi-level coding, trellis coded modulation and space-time trellis coding," IEE Proceedings Communications, vol. 152, no. 6, pp. 1116-1124, Dec 2005. 